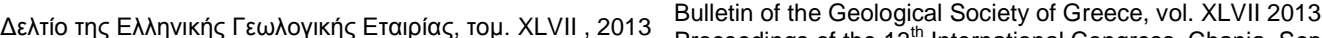

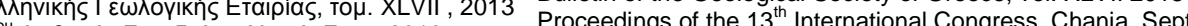

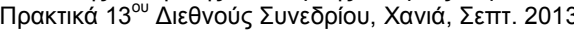
2013

\title{
MAPPING HYDROTHERMAL ALTERATION ZONES FROM SPECTRAL BAND RATIOS: A GEOSTATISTICAL APPROACH BASED ON THE STABLE SEMIVARIOGRAM MODEL
}

\author{
Skianis G. Aim ${ }^{1}$ and Vaiopoulos A. D. ${ }^{2}$ \\ ${ }^{1}$ National and Kapodistrian University of Athens, Faculty of Geology and Geoenvironment, \\ Department of Geography-Climatology, Remote Sensing Laboratory, skianis@geol.uoa.gr \\ ${ }^{2}$ National and Kapodistrian University of Athens, Faculty of Geology and Geoenvironment, \\ Remote Sensing Laboratory
}

\begin{abstract}
An alteration zone may be clearly expressed in an image of a spectral band ratio, as long as the spatial variation of its tonality is strong enough. A measure of the spatial variation of the target of interest (in our case the alteration zone) can be the semivariogram of the band ratio.

In the present paper, the spatial variation of the band ratio, in terms of the statistical behavior of the individual bands, is studied. The semivariograms of the individual bands is simulated by the stable semivariogram model, which is quite flexible in describing the spatial variation of the tonality. The correlation between the spectral bands is also taken into account. Using proper distributions for the reflectance of individual bands and taking into account theorems of statistics, a mathematical expression for the semivariogram of the spectral band is derived. This expression provides information about how the spatial variation of the band ratio is affected by the statistical parameters of the individual bands. Experimentation with satellite images shows that theoretical predictions agree with real data.

The results and conclusions of this paper may help in assessing the efficiency of the images of spectral band ratios over potential mineralization zones.

Key words: Spectral band, band ratio, semivariogram, stable model, alteration zone.
\end{abstract}

\section{Пєрí $\eta \psi \eta$}

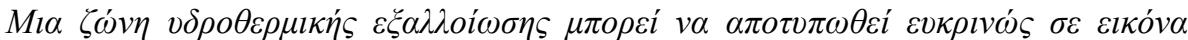

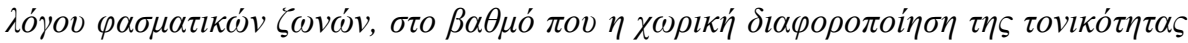

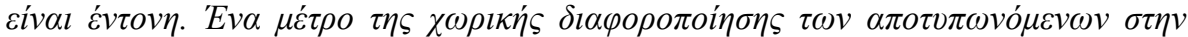

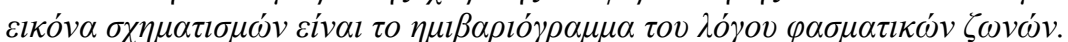

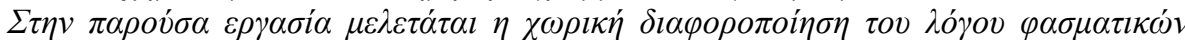

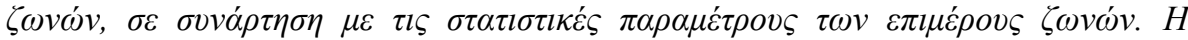

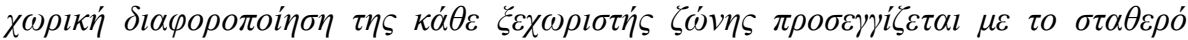

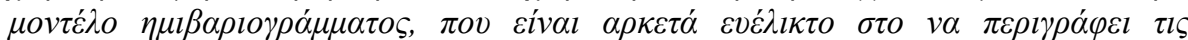

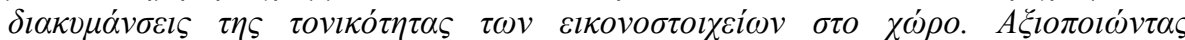

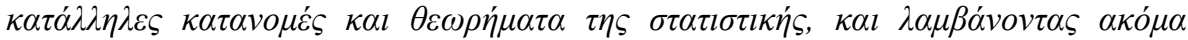

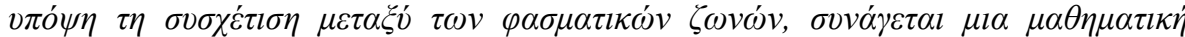

$\underline{\text { XLVII, No } 3-1949}$ 


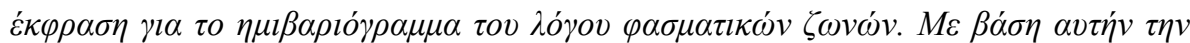

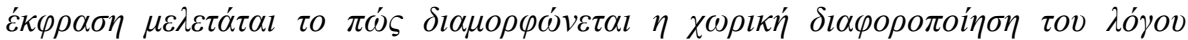

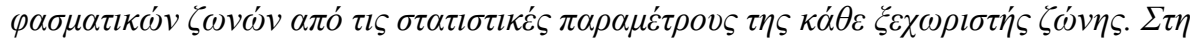

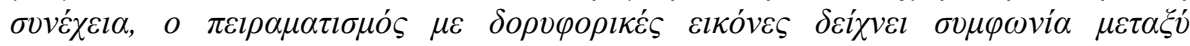

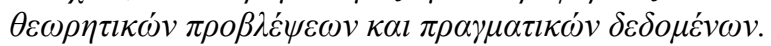

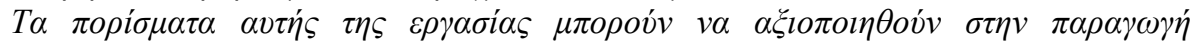

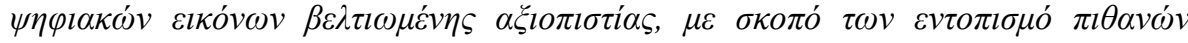
$\mu \varepsilon \tau \alpha \lambda \lambda o \varphi o ́ \rho \omega v \zeta \omega v \omega ́ v$.

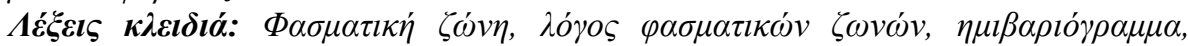

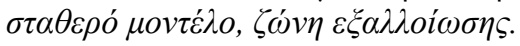

\section{Introduction}

It is well known (Parcharidis et al. 1999; Gupta 2000; Skianis et al. 2012) that the image of the simple spectral band ratio $u$ between two bands with reflectances $x$ and $y$ may be useful in detecting alteration zones, which are potentially associated with hydrothermal mineralization. The ratio $u$ is defined by:

\section{Equation 1 - definition of the band ratio}

$$
u=\frac{x}{y}
$$

$x$ and $y$ may be, for example, the reflectance of band 5 (SWIR 1) and band 7 (SWIR 2) of the Landsat Thematic Mapper, respectively. They can also be reflectances at SWIR band and blue or red band. A reliable measure of the clarity by which the target of interest appears in the band ratio image, can be the intensity of its spatial variation, which may be represented by the semivariogram of the image (Woodcock et al. 1988a, 1988b; Curran 1988; Lacaze et al. 1994; Atkinson 2001; Garrigues et al. 2007).

The semivariogram $\gamma_{x}(h)$ of band $x$ is defined by (Liang 2004):

\section{Equation 2 - definition of the semivariogram}

$$
\gamma_{x}(h)=\frac{1}{2 N} \sum_{i=1}^{N}\left[x\left(p_{i}\right)-x\left(p_{i}+h\right)\right]^{2}
$$

$p_{i}$ is the position of a pixel with a certain reflectance value at band $x \cdot p_{i}+h$ is the position of a pixel at distance $h$ from that at position $p_{i} . N$ is the number of pairs of pixels at distance $h$ each other. In a similar way, the semivariograms $\gamma_{y}(h)$ and $\gamma_{u}(h)$, of band $y$ and ratio $u$, respectively, may be defined.

The definition of the semivariogram, according to equation 2, does not provide much insight about how the spatial variation of the individual bands controls that of the band ratio. This is the subject of the present paper. First, a mathematical expression for the semivariogram $\gamma_{u}(h)$ of the simple band ratio, in terms of the semivariograms of $x$ and $y$, is derived. For such a purpose, proper positively skewed distributions are used in order to model the histograms of bands $x$ and $y$ (Vaiopoulos et. al. 2004) and small variances of $x$ and $y$ are assumed, in order to neglect terms of order higher than one in the Taylor expansion of $u(x, y)$ (Skianis 2012; Skianis \& Vaiopoulos 2012). The semivariograms of $x$ and $y$ are simulated by the stable semivariogram model, the parameters of which may produce a wide variety of spatial variation patterns. Then, the behavior of the expression for $\gamma_{u}(h)$ is studied in order to see how the spatial variation of $u$ is controlled by the parameters of the semivariograms $\gamma_{x}(h)$ and $\gamma_{y}(h)$, as well as by the correlation coefficient 
between $x$ and $y$. Finally, the validity of this mathematical formula is tested by experimentation with satellite images over areas of hydrothermal activity.

\section{The Semivariogram of the Spectral Band Ratio}

The first step to derive a mathematical expression for the semivariogram of the spectral band ratio $\gamma_{u}(h)$ is introduce a semivariogram model to describe the spatial variation of $x$ and $y$ bands. In this paper, the stable model is introduced, which is defined by (Liang 2004):

\section{Equation 3 - the stable semivariogram model}

$$
\gamma_{x}(h)=a\left[1-\exp \left(-\frac{h^{c}}{b^{c}}\right)\right]
$$

$a$ is the sill of the semivariogram and $b$ is the range. Parameter $c$ takes values between 0 and 2 . The same expression can be used to describe $\gamma_{y}(h)$.

In Figure 1 the stable semivariogram model for various values of $c$ is presented. It can be observed that for $c=0, \gamma_{x}(h)\left(\right.$ or $\left.\gamma_{y}(h)\right)$ is a straight horizontal line, which presents a nugget effect at $h=0$ (the semivariogram takes a value which is other than zero). As long as $c$ increases, the convexity of the semivariogram at low $h$ values changes. For $c$ less than unity, the spatial variation of $x$ is relatively strong at small $h$ values, compared to that of $c$ values between 1 and 2 .

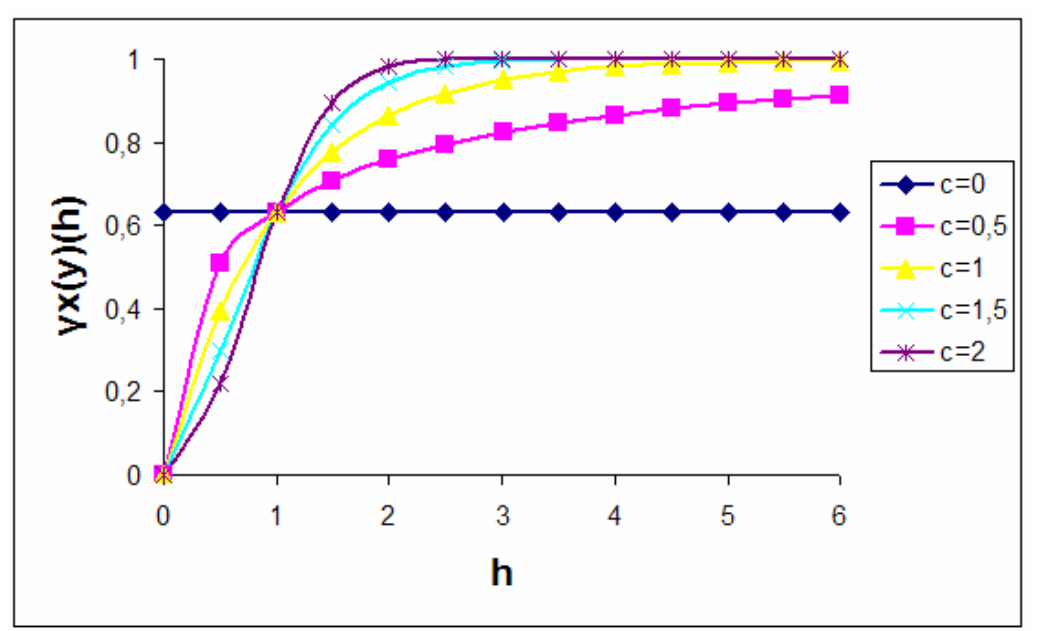

Figure 1 - The stable semivariogram model of $x$ and $y$ bands for various $c$ values. $a=b=1$.

Parameter $c$ provides the semivariogram model with a certain flexibility, in order to describe various types of spatial variation.

Assuming that the variances of $x$ and $y$ are small enough in order to linearize the expression of $u$ in terms of $x$ and $y$, and taking into account a well known theorem of statistics, it can be proved (Skianis 2012, Skianis and Vaiopoulos 2012) that the semivariogram $\gamma_{u}(h)$ of the simple band ratio $u$ is related to $\gamma_{x}(h)$ and $\gamma_{y}(h)$ by:

Equation 4 - the semivariogram $\gamma_{u}(h)$

$\gamma_{u}(h) \cong\left\langle\frac{\partial u}{\partial x}\right\rangle^{2} \gamma_{x}(h)+\left\langle\frac{\partial u}{\partial y}\right\rangle^{2} \gamma_{y}(h)+2 \rho\left\langle\frac{\partial u}{\partial x}\right\rangle\left\langle\frac{\partial u}{\partial y}\right\rangle \sqrt{\gamma_{x}(h) \cdot \gamma_{y}(h)}$

$\rho$ is the correlation coefficient between bands $x$ and $y$. 
The mean values $\langle\ldots>$ can be found, assuming that the histograms of bands $x$ and $y$ may be modeled by simple positively skewed distributions and that mean values $\langle x\rangle$ and $\langle y\rangle$ are constant along the whole images of the spectral bands (Vaiopoulos et. al. 2004, Skianis 2012). Making these necessary calculations and recalling equation (3) for $\gamma_{x}(h)$ and $\gamma_{y}(h)$, equation (4) becomes:

Equation 5 - the final expression for $\gamma_{u}(h)$

$$
\gamma_{u}(h) \cong \frac{\operatorname{Var}(x)}{\operatorname{Var}(y)}\left\{\begin{array}{l}
0,273 \cdot\left[1-\exp \left\{-\left[h / b_{1}\right]^{c_{1}}\right\}+\frac{\pi^{2}}{4} \cdot\left(1-\exp \left\{-\left[h / b_{2}\right]^{c_{2}}\right\}\right)\right]- \\
0,858 \rho \sqrt{\left(1-\exp \left\{-\left[h / b_{1}\right]^{c_{1}}\right\}\right) \cdot\left(1-\exp \left\{-\left[h / b_{2}\right]^{c_{2}}\right\}\right)}
\end{array}\right\}
$$

$\operatorname{Var}(x)$ and $\operatorname{Var}(y)$ are the variances of spectral bands $x$ and $y$, respectively. $b_{1}$ and $b_{2}$ are the ranges of the semivariograms of $x$ and $y$, respectively. $c_{1}$ and $c_{2}$ are the values of $c$ for the semivariograms of $x$ and $y$, respectively.

Equation (5) provides the theoretical background to study the spatial variation of the simple band ratio $u$, in terms of the spatial variation of the spectral bands $x$ and $y$. It is obvious that the sill of the semivariogram $\gamma_{u}(h)$ depends on the ratio $\operatorname{Var}(x) / \operatorname{Var}(y)$, as well as on the correlation coefficient $\rho$ between the spectral bands $x$ and $y$.

In Figure 2, it can be seen how the form of the semivariograms $x$ and $y$, which is controlled by the parameter $c$, influences the behavior of $\gamma_{u}(h)$. For $c=0$, a horizontal semivariogram curve is produced. For $c=2$, the curve $\gamma_{u}(h)$ is convex for small values of $h$. For $c=1$, the curve $\gamma_{u}(h)$ is concave.

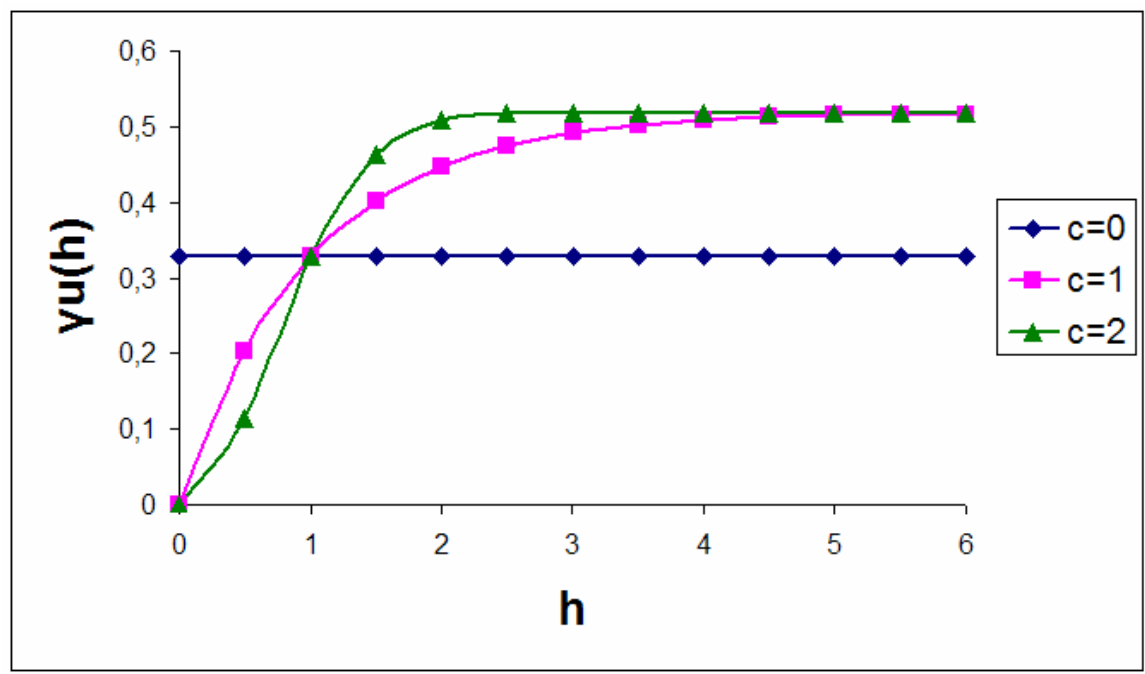

Figure 2 - The semivariogram of $u$ for various values of $c$, which is common for the semivariograms of $x$ and $y \cdot \operatorname{Var}(x)=\operatorname{Var}(y), \rho=0,5, b_{1}=b_{2}=1$.

In Figure 3 and Figure 4, it can be seen how the parameters $c_{1}$ and $c_{2}$ influence the shape of $\gamma_{u}(h)$, for various values of the correlation coefficient $\rho$. For $c_{1}=1$ and $c_{2}=2$ and small $h$ values, the curve $\gamma_{u}(h)$ is convex. On the other hand, the curve is concave for $c_{1}=2$ and $c_{2}=1$. The sill of the semivariogram of $u$ decreases, as long as $\rho$ increases.

In Figure 5 it can be seen how different $c_{1}$ and $c_{2}$ values produce semivariograms $\gamma_{u}(h)$ with different characteristics. When $c_{1}$ or $c_{2}$ is equal to zero, a nugget effect is produced at $h=0$. For $c_{1}$ and $c_{2}$ different from zero, the curve $\gamma_{u}(h)$ starts from the origin of the axes. $\gamma_{u}(h)$ is convex at small $h$ values, for $c_{2}=2$ and $c_{1}$ equal to zero or unity. In such a case the semivariogram $\gamma_{u}(h)$ 
reaches the sill at lower $h$ values than a concave semivariogram, like that of $c_{1}=2$ and $c_{2}=1$ does.

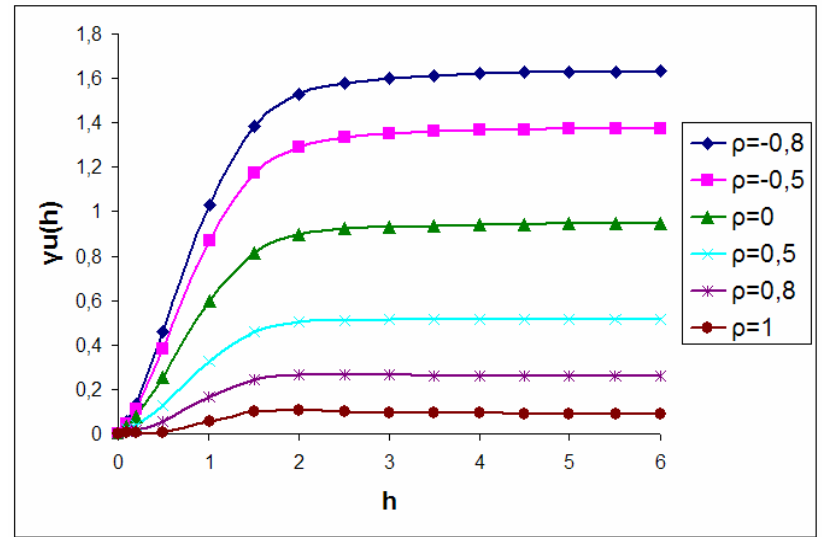

Figure 3 - The semivariogram of $u$ for $c_{1}=1$ and $c_{2}=2 . \operatorname{Var}(x)=\operatorname{Var}(y), b_{1}=b_{2}=1$.

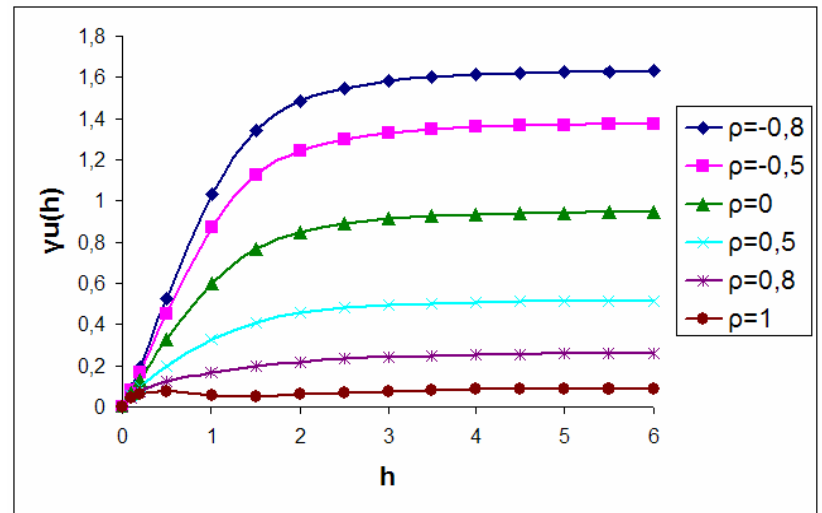

Figure 4 - The semivariogram of $u$ for $c_{1}=2$ and $c_{2}=1 . \operatorname{Var}(x)=\operatorname{Var}(y), b_{1}=b_{2}=1$.

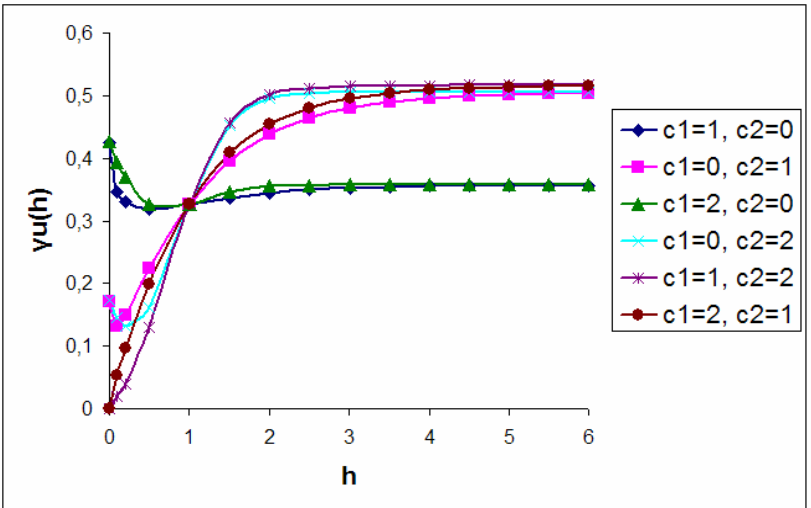

Figure 5 - Semivariograms $\gamma_{u}(h)$ for various $c_{1}$ and $c_{2}$ values. $\operatorname{Var}(x)=\operatorname{Var}(y), \rho=0,5, b_{1}=b_{2}=1$.

\section{Methodology of Experimentation with a Multispectral Image}

One of the main objectives of the present study is to examine and compare the actual semivariograms of satellite imagery (depicting hydrothermal activity and/or mineralization), with the theoretical semivariograms, which can be calculated from equations 3 and 5 . The scope of the above objective is to observe and measure how well the theoretical models can fit and predict the real data. 
As stated in the introduction, the semivariograms of interest are those of band 5 and band 7 of the Landsat 7 satellite image, as well as the spectral band ratio band5/band7, which can provide valuable information for tracking and mapping hydroxyl-bearing minerals which are related with hydrothermal activity (Parcharidis et. al. 2001, Bodruddoza and Fujimitsu 2012)

Specifically, the methodology in order to obtain the theoretical semivariogram of the band ratio, is described below:

1. First of all, Landsat-7 ETM+ imagery over Milos Island, Aegean Sea, is acquired. It is well known that hydrothermal activity and mineralization is developed in this area (Naden et al. 2003).

2. Secondly, the actual semivariograms of bands 5 and 7 are calculated, using equation (2).

3. Then, the actual semivariogram of band ratio $5 / 7$ is calculated, using the same equation. The calculations of step 1 and step 2, as well as the calculation of the correlation coefficient between the spectral bands, were performed with a Matlab program, which was developed for the purpose of the present study.

4. Afterwards, the theoretical semivariograms of bands 5 and 7 are estimated, assuming that the actual semivariogram can be approximated with proper calibration of the stable semivariogram model. This model is expressed in equation 3. Parameters $a$ and $b$ (sill and range respectively), are now known, since they can be obtained from the previously calculated actual semivariograms of step 2. Parameter $c$ needs manual adjustment and experimentation for fine tuning. The value of $c$, which yields the best approximation of the actual semivariance curve, is kept.

5. Calculation of the correlation coefficient $(\rho)$ between the two bands $(5,7)$ is carried out.

6. Finally, the theoretical semivariogram of the band ratio 5/7 can be calculated, by applying the equation 5 .

At this point every parameter is known. $\operatorname{Var}(x)$ and $\operatorname{Var}(y)$ are the sills of the actual semivariograms of band 5 and 7 respectively, calculated in step 2. Parameters $b_{1}$ and $b_{2}$ are also known from step 2 (range of band 5 and 7 respectively). Parameters $c_{1}$ and $c_{2}$ are determined in step 4, by a trial and error procedure. The correlation coefficient $(\rho)$ is known from step 5.

\section{Area of Interest - Milos Island}

In Figure 6, the satellite image of Milos Island is presented. At the eastern part of the island, hydrothermal activity is developed (Fontaine et al. 2003), therefore it is interesting to map the band ratio 5/7, over this area. In Figures 7 and 8, the channels 5 and 7, respectively, over the eastern part of the island, are presented. In Figure 9, the band ratio 5/7 is mapped.

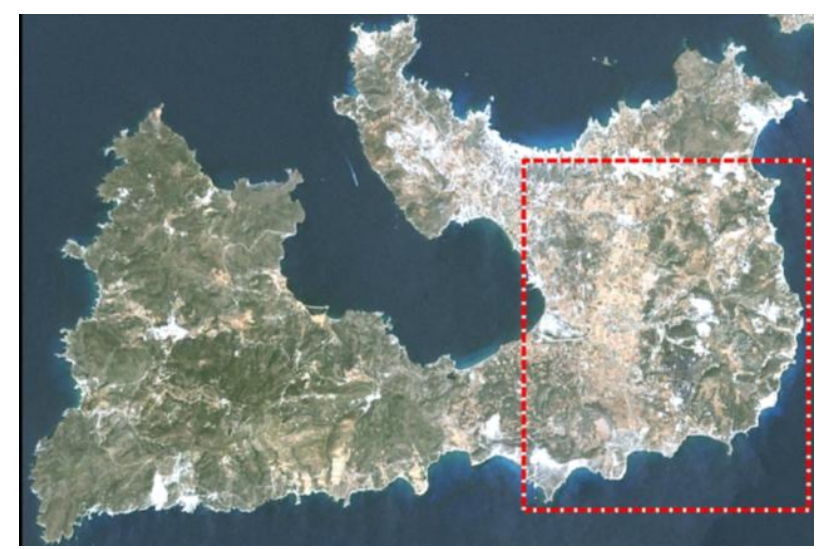

Figure 6 - A Landsat-7 natural color composite (321) of Milos island. Hydrothermal manifestations are present in the studied red framed area, among other sites in the island.

XLVII, No 3 - 1954 

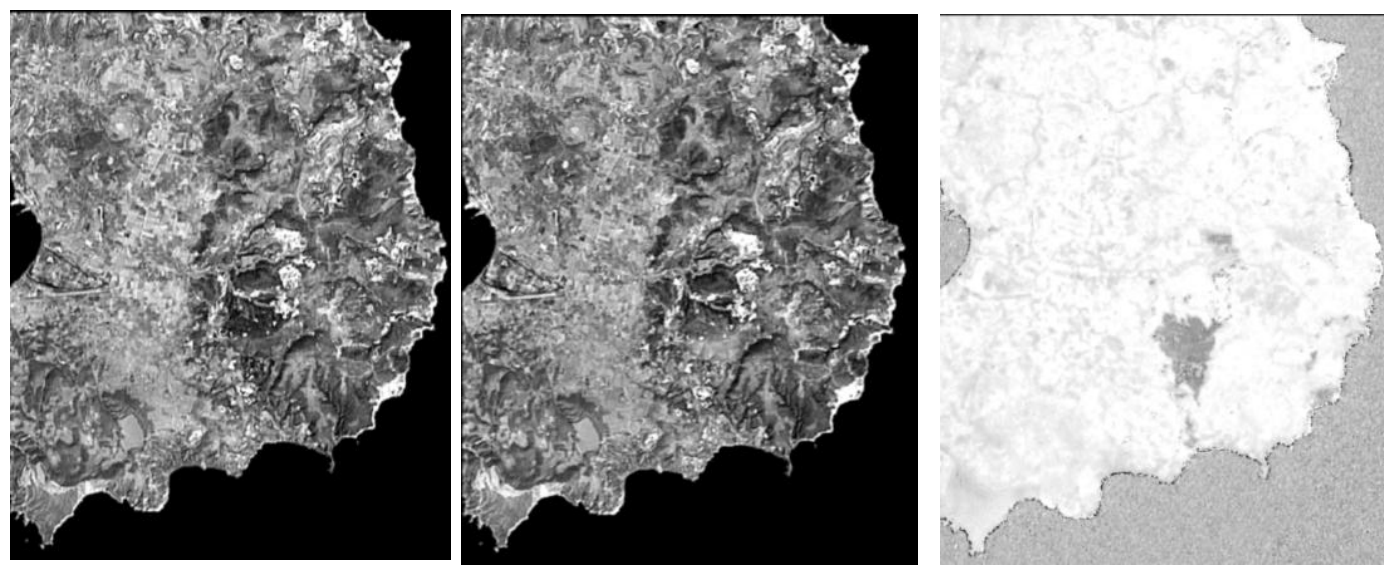

Figure 7,8 and 9 (Left to right) - Visualization of band 5, band 7 and ratio 5/7 respectively. The images above are depicting the red framed area of figure 6.

\section{Results}

In Figures 10, 11 and 12 the semivariograms of bands 5 and 7, as well as those of the ratio 5/7 are presented. It must be noted that in the above graphs, $y$-axis measures semivariance in square brightness values of the image, while $\mathrm{x}$-axis measures distance $(h)$ in pixels. Since the imagery has been pan sharpened from Landsat-7 panchromatic band with the HPF method (Nikolakopoulos et al. 2010, Vaiopoulos 2011), distances must be multiplied by 15, in order to be converted to meters.

Bands 5 and 7 have a very high correlation coefficient, equal to 0.9843 .

The horizontal semivariogram of band 5 has been fitted by a stable semivariogram model with $a_{1}=$ $3200, b_{1}=80, c_{1}=0.8$. The vertical semivariogram of band 5 has been fitted by $a_{1}=4000, b_{1}=80$, $c_{1}=1.0$. The parameters of the stable model which fits the horizontal semivariogram of band 7 are $a_{2}=2500, b_{2}=250, c_{2}=0.8$. Finally, the parameters of the stable model for the vertical semivariogram of band 7 are $a_{2}=2500, b_{2}=300, c_{2}=0.6$.

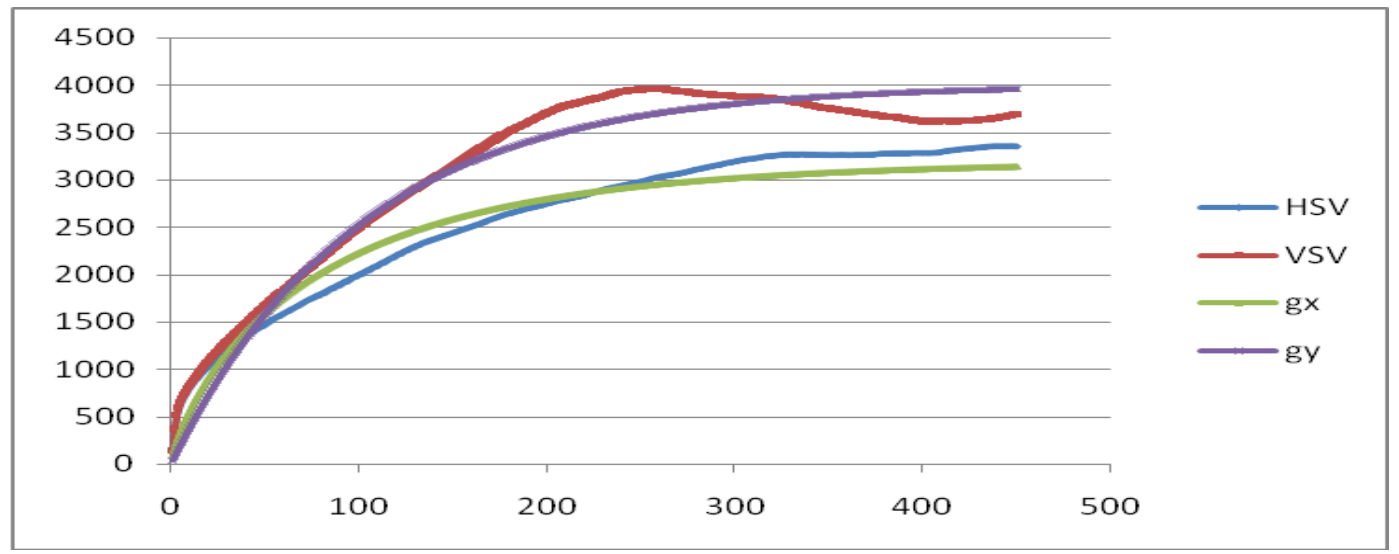

Figure 10 - Actual Horizontal and Vertical semivariograms (HSV, VSV) and theoretical horizontal and vertical semivariograms (gx, gy) of band 5. 


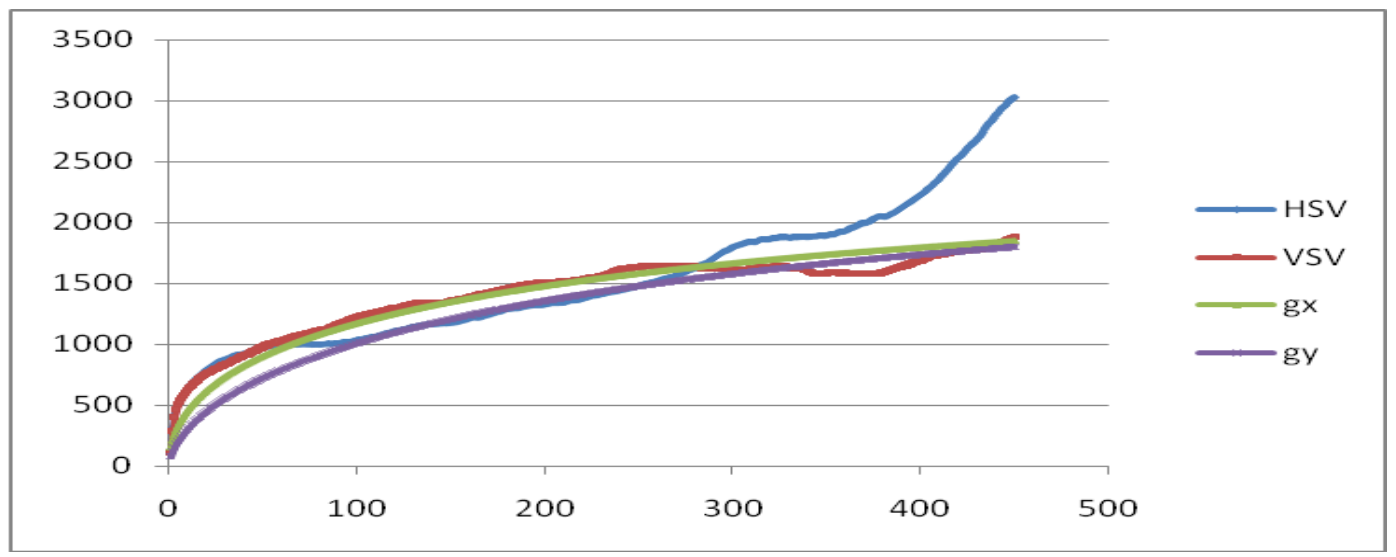

Figure 11 - Actual Horizontal and Vertical semivariograms (HSV, VSV) and theoretical horizontal and vertical semivariograms (gx, gy) of band 7.

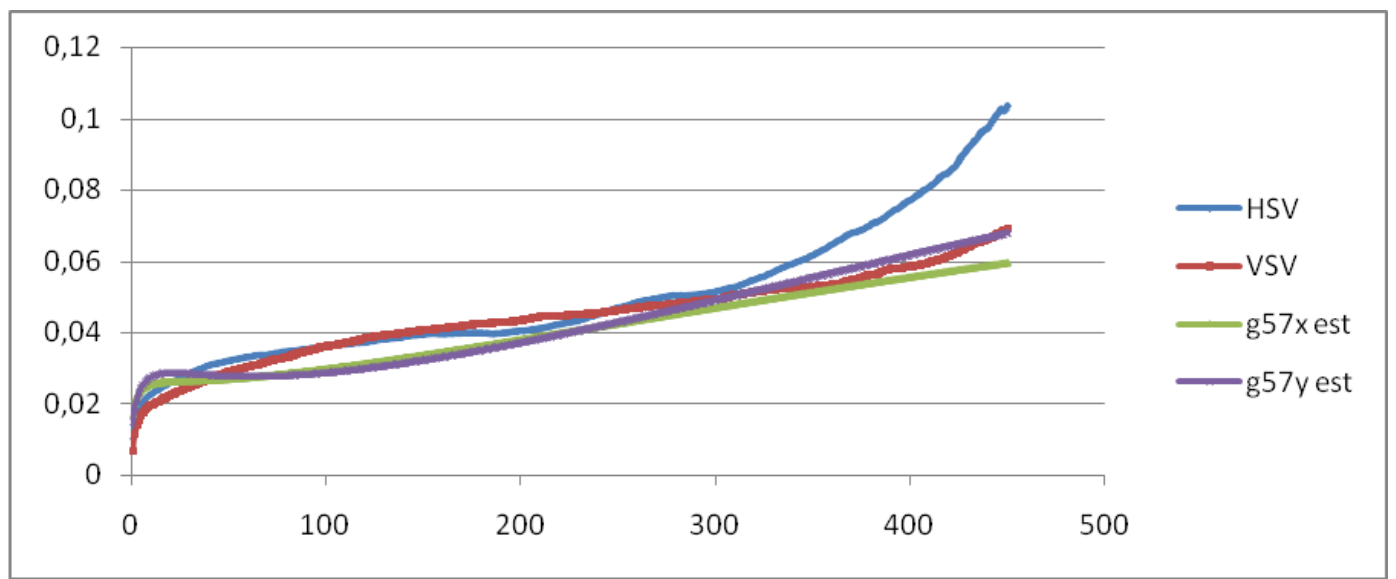

Figure 12 - Actual Horizontal and Vertical semivariograms (HSV, VSV) and theoretical horizontal and vertical semivariograms (g57x est, g57y est) of band ratio 5/7.

From the diagrams of Figures 10 and 11, it can be observed that the stable semivariogram model (equation 3) provides satisfactory approximation of the actual semivariograms for distance $\sim 400$ pixels (or $6 \mathrm{~km}$ ). A notable exception is the actual horizontal semivariogram of band 7 (see Figure 11), which presents a strong ascending tendency for $h$ more than 300 pixels (or $4.5 \mathrm{~km}$ ). This curve should be fitted by a more complex semivariogram model, or by a combination of standard semivariogram models. However, fitting of complex semivariograms is beyond the scope of the present paper. Anyway, with the exception of the actual horizontal semivariogram of band 7, the theoretical models seem to approximate well the actual semivariograms.

In addition, as it can be observed in Figure 12, the theoretically calculated vertical semivariogram according to equation 5 , is in good agreement with the actual semivariogram. Considerable deviations between theory and actual data are observed in the case of the horizontal semivariogram $5 / 7$, since the stable model could not fit adequately the horizontal semivariogram of band 7 .

\section{Discussion and Conclusions}

Equation (5) gives an insight on how the spatial variation of the individual spectral bands controls the semivariogram of the band ratio. The ratio $\operatorname{Var}(x) / \operatorname{Var}(y)$ of the variances (sills) of bands $x$ and $y$ and the correlation coefficient $\rho$ between the two bands control the sill of the semivariogram 
$\gamma_{u}(h)$ of $x / y$. A high $\operatorname{Var}(x) / \operatorname{Var}(y)$ value favors the production of a semivariogram $\gamma_{u}(h)$ with a high sill. On the contrary, a high correlation coefficient $\rho$ produces a $\gamma_{u}(h)$ with a low sill.

The parameter $c$ controls the convexity of the semivariogram $\gamma_{u}(h)$ at low $h$ values. More specifically, when the parameter $c_{2}$ of the semivariogram of $y$ is equal or tends to 2 , the curve $\gamma_{u}(h)$ is convex at low $h$ values, which means that the spatial variation of the spectral ratio $x / y$ is relatively smooth for small distances $h$ between pixels. On the other hand, for $c_{2}$ more than zero and not exceeding unity, the curve $\gamma_{u}(h)$ is concave, therefore a relatively strong spatial variation between pixels at small distances $h$ is developed.

The physical meaning of these comments is that in order to produce an image of a spectral band ratio with clearly expressed targets of interest, such as hydrothermal zones, a high ratio $\operatorname{Var}(x) / \operatorname{Var}(y)$ and a low correlation coefficient between $x$ and $y$ is needed. A low $c_{2}$ value of the semivariogram of $y$ (which actually means a concave semivariogram), also favors the production of a $x / y$ image with a good spatial variation.

The bands 5 and 7 of the multispectral Landsat ETM+ image of Milos island are very strongly correlated. This is an important reason why the band ratio of Figure 6 has a diffuse bright tonality, which does not help in discriminating different targets of interest. On the other hand, as long as the individual bands 5 and 7 are adequately fitted by the stable semivariogram model, the theoretically calculated semivariogram of the ratio 5/7 is in a good agreement with the actual one.

The results and conclusions of this paper may be useful in mapping hydrothermal zones with the aid of multispectral imagery, in the context of a mineral exploration project.

\section{References}

Atkinson P.M. 2001. Geostatistical regularization in remote sensing. In Atkinson, N. J. and Atkinson, P. M. (eds), Modelling Scale in Geographic Information Science, 237-260, Wiley, New York.

Bodruddoza M. and Fujimitsu Y. 2012. Mapping hydrothermal altered mineral deposits using Landsat 7 ETM+ image in and around Kuju volcano, Kyushu, Japan, Journal of Earth System Science, 121(4), 1049-1057.

Curran P.J. 1988. The semivariogram in remote sensing: An introduction, Remote Sens. Environ., 24(3), 493-507.

Fontaine F.Jh., Rabinowicz M. and Boulegue J. 2003. Hydrothermal processes at Milos Island (Greek Cyclades) and the mechanisms of compaction-induced phreatic eruptions, Earth and Planetary Science Letters, 210 (1-2), 17-33.

Garrigues S., Allard D. and Baret F. 2007. Using First- and Second -Order Variograms for Characterizing Landscape Spatial Structures From Remote Sensing Imagery, IEEE Transactions on Geoscience and Remote Sensing, 45(6), 1823-1834.

Gupta R.P. 2003. Remote Sensing Geology, second edition, Berlin, Heidelberg. Springer-Verlag, $655 \mathrm{pp}$.

Lacaze B., Rambal S. and Winkel T. 1994. Identifying spatial patterns of Mediterranean landscapes from geostatistical analysis of remotely sensed data, Int. J. Remote Sens, 15(12), 2437-2450.

Liang S. 2004. Quantitative Remote Sensing of Land Surfaces, Wiley-Interscience, New York, 534 pp.

Naden J., Kilias S.P., Leng M.J., Cheliotis I. and Shepherd T.J. 2003. Do fluid inclusions preserve $\delta 18 \mathrm{O}$ values of hydrothermal fluids inepithermal systems over geological time? Evidence from paleo- and modern geothermal systems, Milos island, Aegean Sea, Chemical Geology $197,143-159$

Nikolakopoulos K.G., Vaiopoulos A.D. and Tsobos P.I. 2010. Ameliorating the spatial resolution of GeoEye data, Proc. SPIE 7831, Earth Resources and Environmental Remote Sensing/GIS Applications, 783104, doi:10.1117/12.864442.

XLVII, No 3 - 1957 
Parcharidis I., Gartzos E. and Psomiadis E. 1999. Alteration zones detection in Lesbos island (Greece), through the application of Landsat 5 TM band ratio images, Journal of Mineral Wealth 112, 37-46.

Parcharidis I., Gartzos E. and Psomiadis E. 2001. Evaluation of different remote sensing methods for the detection of hydrothermal alteration zones in Milos island (Greece). Proceedings of the $9^{\text {th }}$ International Congress of the Geological Society of Greece, Vol. 5, 2047-2054.

Skianis G. Aim. and Vaiopoulos A. D. 2012. The spatial variation of a spectral band ratio with a considerable correlation between the bands, Proceedings of the $4^{\text {th }}$ EARSel Workshop on Remote Sensing and Geology, Mykonos, Greece, 24th-25th May 2012, 28-43.

Skianis G. Aim. 2012. The problem of the spatial variation of a spectral band ratio: a first order approach based on probability theory, Earth Science Informatics, 5(1), 13-21.

Skianis G. Aim., Nikolakopoulos K. G. and Vaiopoulos D. A. 2012. Remote Sensing, Athens, Ion publications, $336 \mathrm{pp}$.

Vaiopoulos A.D. 2011. Developing Matlab scripts for image analysis and quality assessment, Proc. SPIE 8181, Earth Resources and Environmental Remote Sensing/GIS Applications II, 81810B, doi:10.1117/12.897806.

Vaiopoulos D. A., Skianis G. Aim. and Nikolakopoulos K. 2004. The contribution of probability theory in assessing the efficiency of two frequently used vegetation indices, International Journal of Remote Sensing, 25(20), 4219-4236.

Woodcock C.E., Strahler A. and Jupp D.L.B, 1988a. The use of variograms in remote sensing: A/scene models and simulated images, Remote Sens. Environ, 25(3), 323-348.

Woodcock C.E, Strahler A.H. and Jupp D.L.B. 1988b. The use of variograms in remote sensing: B/Real digital images, Remote Sens. Environ, 25(3), 349-379. 